\title{
L'HOMME L'Homme
}

Revue française d'anthropologie

$221 \mid 2017$

Varia

\section{Georges Balandier (1920-2016)}

\section{Emmanuel Terray}

\section{OpenEdition}

Journals

Édition électronique

URL : http://journals.openedition.org/lhomme/30082

DOI : 10.4000/lhomme.30082

ISSN : 1953-8103

\section{Éditeur}

Éditions de l'EHESS

\section{Édition imprimée}

Date de publication : 1 mars 2017

Pagination : 5-10

ISBN : 978-2-7132-2688-5

ISSN : 0439-4216

Référence électronique

Emmanuel Terray, «Georges Balandier (1920-2016) », L'Homme [En ligne], 221 | 2017, mis en ligne le 01 mars 2017, consulté le 16 février 2021. URL : http://journals.openedition.org/lhomme/30082 ;

DOI : https://doi.org/10.4000//homme.30082 


\title{
Georges Balandier (1920-2016)
}

\author{
Emmanuel Terray
}

Au commencement de cet automne ensoleillé de 2016, Georges Balandier nous a quittés. En ce premier moment du deuil, je laisserai à d'autres, plus jeunes et plus compétents que moi, la tâche de mesurer l'importance de l'œuvre, la richesse et la diversité de l'apport scientifique, la profondeur de l'influence exercée. J'essaierai simplement de dire ce que Georges Balandier a été pour nous - nous: la génération des anthropologues africanistes des années 1960 -, la place qu'il a tenue dans nos vies, la dette que nous avons contractée envers lui. Je n'ai nulle qualité pour parler au nom d'autrui, et je n'évoquerai que mon cas personnel; mais comme mon parcours n'a rien d'original, je crois que beaucoup pourront s'y reconnaître.

J'ai raconté ailleurs comment j'en suis venu à rencontrer Georges Balandier et à me ranger "sous sa bannière ». Entré à l'École normale en 1956, j'avais entrepris des études de philosophie, mais j'avais bientôt compris que celles-ci me conduiraient à une existence confinée entre les bibliothèques et les salles de cours; j'aspirais à davantage d'espace et de mouvement. C'était par ailleurs le temps de la guerre d'Algérie, où la France offrait un visage parfois hideux, et je désirais "prendre le large». Je me tournai donc vers une discipline qui, à tout le moins, me promettait voyages et dépaysements : l'anthropologie.

L'anthropologie d'alors était marquée par l'entreprise structuraliste et par l'œuvre de Claude Lévi-Strauss. Comme tant d'autres, j'ai été fasciné par la lecture de Tristes Tropiques, et j'ai été émerveillé par la performance intellectuelle que représentent Les Structures élémentaires de la parenté. Mais rejoindre la cohorte des élèves de Claude Lévi-Strauss, c'était se diriger vers des sociétés océaniennes ou amérindiennes bien éloignées de la 
"grande scène » de l'histoire: mes passions militantes n'y trouvaient pas leur compte. Restait l'Afrique; ce qu'il est convenu d'appeler l'école de Griaule occupait alors le terrain. Elle me semblait tout entière absorbée par l'étude des systèmes de représentation des cosmogonies, des mythes et des contes; du coup, elle privilégiait la parole de quelques spécialistes et laissait la masse de côté; de la même façon, le discours l'intéressait davantage que la pratique, et elle me paraissait indifférente à l'existence quotidienne, aux dures réalités du travail, de la pauvreté et de la sujétion.

"Alors Balandier vint... ${ }^{1}$. Il avait déjà publié quatre de ses œuvres majeures: Sociologie actuelle de l'Afrique noire $(1955)^{2}$, Sociologie des Brazzavilles noires $(1955)^{3}$, La Vie quotidienne au royaume de Kongo $(1965)^{4}$ et Afrique ambiguë $(1957)^{5}$. Soucieux de recruter de nouveaux adeptes, il tint dès 1959 un séminaire à l'École normale; avec Pierre Bonnafé et Marc Augé, je fus très séduit, et ce fut le début d'un long cheminement sous sa bienveillante tutelle.

Qu'y avait-il de si attirant dans le propos de Georges Balandier? En premier lieu, l'accent placé sur la situation coloniale. La notion est devenue aujourd'hui banale; elle ne l'était nullement à l'époque : sourde aux mouvements du monde, la France de 1950 vivait encore très largement dans le pieux souvenir de son "empire» et de sa "mission civilisatrice». Or, voici qu'étaient mises au jour les vérités cruelles de la domination coloniale : les travaux forcés, les corvées, les taxes, les recrutements, bref, les mille et un procédés de l'exploitation; mais aussi, et peut-être surtout, le mépris et les humiliations, sans oublier ce qui fut probablement la pire d'entre elles : un paternalisme non moins infantilisant que balourd.

Face à l'hydre coloniale, Georges Balandier découvrait également la résistance africaine. Il dira plus tard que son engagement de 1943-1944 dans le maquis a joué un rôle décisif pour fonder son refus catégorique de toutes les formes de l'asservissement et sa solidarité indéfectible avec toutes les modalités de la protestation. On se souvient de sa description des messianismes congolais : en un temps où les Africains colonisés se voient

1. Cf. Emmanuel Terray, "Présentation", in Afrique plurielle, Afrique actuelle. Hommage à Georges Balandier, Paris, Karthala, 1986: 10.

2. Sociologie actuelle de l'Afrique noire. Dynamique des changements sociaux en Afrique centrale. Paris, Presses universitaires de France, 1955 ("Bibliothèque de sociologie comparative»).

3. Paris, Armand Colin, 1955 ( Cahiers de la Fondation nationale des sciences politiques» 67).

4. La Vie quotidienne au royaume de Kongo, du XVI au XVIII siècle. Paris, Hachette, 1965 ("La Vie quotidienne").

5. Paris, Plon, 1957 («Terre humaine»). 
interdire toute expression politique autonome, les messianismes représentent une voie détournée par laquelle, sous les dehors d'une prédication religieuse, des exigences clairement politiques - la liberté, l'indépendance se font entendre. Bien loin de constituer une masse inerte, les Africains s'affirment donc comme acteurs de leur propre destin. De même, dans son étude des Brazzavilles noires, Georges Balandier montre comment les villes africaines sont le creuset d'une Afrique nouvelle, de part en part ouverte aux souffles de la modernité.

À travers ces analyses, c'est une autre anthropologie qui s'impose. Georges Balandier la déclare "dynamique et critique». Sa caractéristique première est, à mon sens, de faire toute sa place à l'histoire: toutes les sociétés, qu'elles le veuillent ou non, qu'elles s'en défendent ou non, sont dans l'histoire; toutes sont confrontées à leur propre usure au-dedans, et aux menaces de toutes natures qui peuvent peser sur elles du dehors; toutes connaissent à l'occasion les crises et le désordre; toutes élaborent les dispositifs et les stratégies qui leur permettent de maîtriser l'événement et de concilier continuités et changements. De plus, sur la longue durée, il n'y a pas de société apaisée ou pacifiée ; toutes les sociétés sont déchirées par des contradictions et par les conflits qui en sont l'effet : entre les sexes, entre les générations, entre les territoires, entre les groupes professionnels - paysans, éleveurs, artisans -, entre les groupes statutaires - nobles, hommes libres, esclaves. Partout, ouvert ou latent, on rencontre l'antagonisme, qui est la chance de l'anthropologue dans la mesure où il est un excellent révélateur des structures cachées de la société.

Bien entendu, il serait inexact de considérer le Balandier de cette époque comme un astre isolé. Au même moment, les chercheurs britanniques de l'école dite de Manchester, et en particulier Max Gluckman, développent des orientations très voisines, et c'est par l'intermédiaire de Georges Balandier que nous aurons accès à leurs travaux. On peut également s'interroger sur le rapport que les uns et les autres ont entretenu avec le marxisme. On sait que Max Gluckman fut longtemps membre du parti communiste sud-africain ; de son côté, Georges Balandier a publié, en 1949, dans la Revue socialiste, un article intitulé "Marxisme et ethnologie», dans lequel l'apport marxiste est examiné avec une indéniable sympathie. En fait, aucun des deux ne se soucie de la philosophie matérialiste; en revanche, le «motif» de la lutte des classes, avec ce qu'il implique d'attention portée à la contradiction et au conflit, ne peut que retenir leur intérêt.

À ma connaissance, Georges Balandier s'est toujours tenu à l'écart de la politique partisane, mais il a su s'engager résolument au service des causes qui lui étaient chères : c'est ainsi qu'il prendra une part active, aux côtés d'Alioune Diop et de bien d'autres, à l'aventure de Présence africaine; 
manière pour lui de contribuer à la libération culturelle et intellectuelle de l'Afrique, sans laquelle les indépendances risquaient fort, à ses yeux, de n'être qu'un leurre.

Quelle a été l'influence de Georges Balandier? Il suffit, pour en mesurer l'importance, de consulter la liste de ceux qui furent, d'une façon ou d'une autre, ses élèves. J'ai déjà cité Pierre Bonnafé et Marc Augé, auxquels il faut ajouter Michel Izard, Michel Cartry, Alfred Adler, Jean Bazin, Jean-Loup Amselle, Claudine Vidal, Marc Le Pape, Jean-Marie Gibbal, Alain Marie, Pierre-Philippe Rey, Georges Dupré, Jean-Pierre Dozon, Alfred Schwartz, Claude Meillassoux et bien d'autres encore qui me pardonneront de ne pas les nommer. À vrai dire, y a-t-il eu durant ces années un seul africaniste français qui n'ait pas été peu ou prou touché par le rayonnement de Georges Balandier?

De plus, le nombre n'est pas ici l'élément principal. Ce qui importe, c'est le rapport très particulier que Georges Balandier a su entretenir avec ses élèves. Pour le dire d'un mot, il a toujours été extraordinairement soucieux de leur sort et attentif à leurs problèmes. Je ne parle pas seulement des problèmes théoriques de la recherche; les jeunes chercheurs ont besoin de postes stables qui leur permettent de se consacrer entièrement à leurs travaux : il leur faut des crédits de mission, des affectations sur le terrain, etc. Georges Balandier a dépensé sans compter son temps et son énergie pour leur procurer tout cela. On a parfois raillé le caractère un peu boulimique de ses engagements dans les institutions: la Sorbonne, l'EHESS, l'Orstom (devenu l'IRD), la Fondation nationale des sciences politiques... En réalité, il s'agissait pour lui d'occuper des positions à partir desquelles il pourrait venir efficacement en aide à ses élèves. Pour m’en tenir à un seul exemple, c'est ainsi que Michel Cartry, Pierre Bonnafé et moi, nous nous sommes succédé à la Fondation nationale des sciences politiques en attendant de partir pour l'Afrique. J'ajoute - pour en avoir fait personnellement l'épreuve - que lorsque l'un d'entre nous se trouvait ou se plaçait en situation (politiquement ou administrativement) difficile, l'appui de Georges Balandier ne lui faisait jamais défaut; il ne tirait jamais argument de nos imprudences ou de nos témérités pour nous retirer son soutien.

Sur le plan proprement intellectuel, Georges Balandier reste dans mon souvenir comme un maitre à la fois infiniment tolérant et remarquablement exigeant. Infiniment tolérant: il n'imposait aucune "orientation" déterminée, et il me suffira de citer les noms de Claude Meillassoux et de Michel Cartry pour marquer l'extrême diversité des tendances auxquelles il fut confronté. Chacun restait libre de ses choix. En revanche, une fois ces choix effectués, il fallait les assumer avec rigueur et conséquence, et c'est alors que Georges Balandier devenait exigeant, pourchassant 
inlassablement les naïvetés, les préjugés, les inconséquences et les équivoques. Autrement dit, nous étions maîtres de nos idées, mais il fallait être capable de les défendre. Qu'on me permette une fois encore d'évoquer mon cas; j'ai préparé sous sa direction ma thèse sur l'histoire du royaume abron; pour parler comme Thucydide, les conseils et les critiques qu'il m'a prodigués tout au long de ce travail restent dans mon esprit comme "un trésor pour toujours".

Dans le petit livret que la famille de Georges Balandier a fait distribuer le jour de ses obsèques, je trouve un texte de lui qui le dépeint très fidèlement, dans ce qu'il voulut être comme dans ce qu'il fut :

"Si j'étais lié à un panthéon africain, je choisirais comme divinité, comme figure à imiter ou à l'intérieur de laquelle m’installer, Legba. C'est le dieu des carrefours, de la communication, des seuils, des passages. Il est présenté comme nécessaire aux autres dieux qui ont, par fonction, un pouvoir supérieur au sien quant à la création et l'ordre du monde. Mais sans lui, ils sont impuissants.

Favoriser les relations, faire circuler du sens, n'est-ce pas le plus beau rôle? " ${ }^{6}$

Adieu Georges Balandier, ou plutôt au revoir, car même si vous êtes maintenant entré dans l'invisible, votre mémoire et votre œuvre demeurent parmi nous, et par leur intermédiaire vous n'avez pas fini de favoriser les relations et de faire circuler du sens.

École des hautes études en sciences sociales, Paris

6. Cf. Xavier Lacavalerie \& Michèle Gazier, «L'anthropologue Georges Balandier, spécialiste de l'Afrique, est mort ", Télérama.fr, 5 octobre 2016 [http ://www.telerama.fr/livre/l-anthropologuegeorges-balandier-specialiste-de-l-afrique-est-mort,148357.php]. 
